\title{
3D thermal field modelling in electromagnetic gripping system
}

\author{
Mariusz Barański ${ }^{1,}$, Krystian Glapa ${ }^{2}$ \\ ${ }^{1}$ Politechnika Poznańska \\ ${ }^{2}$ Krotoszyn
}

\begin{abstract}
In this paper, 3D steady-state thermal field modeling in electromagnetic gripping system using Comsol Multiphisics was presented. The electromagnetic gripping system, which is a component of the mechanical leg of a walking robot was designed by the authors. An algorithm to design of the electromagnetic gripping was developed. During calculations, the influence of the value of the current on the thermal field distribution in steady-state was carried out. Selected results of simulations as well as the analysis of these results were presented.
\end{abstract}

\section{Introduction}

Gripping devices are currently the most varied in design components of robots and manipulators, due to the increasing use of them in many branches of industry and the diversity of manipulated objects. One of the popular types of such devices are electromagnetic grippers. They are widely used both in manipulation systems as well as in mobile robots walking mechanisms $[1,2,3$, 4]. The global industry enforces the need of design and develop new constructions of gripping systems. In new designs of this devices the constructors are looking for new materials, including materials that will better dissipate heat [5]. The use of them makes it possible to obtain the same nominal parameters of device at a much smaller size of the unit [3]. Then the system has a higher power density.

The dynamic evolution of computer technology allows the reduction of costs, related to building many prototypes. The commonly used computer modeling causing the replacement of traditional analytic models with numeric ones. Computer analyzes, including heat transfer problems, are carrying out to provide the most accurate mapping of phenomena occurring in electromagnetic devices $[9,10]$. To analyze them, advanced professional software is used, such as Ansys, Infolytica or Comsol Multiphysics [6,7]. In this paper the Comsol environment been used, which uses Finite Elements Method (FEM) to solve partial differential equations [7].

\section{The design algorithm and modeling process of the electromagnetic gripper}

In this paper was assumed that the designed gripper, will be the component of mobile robot's leg. The robot will weigh $100 \mathrm{~kg}$, and he can carry $25 \mathrm{~kg}$ of equipment. The force of gravity acting on the system is $1600 \mathrm{~N}$. To ensure safe operation, in example when the robot encounters a curved surface, non-homogenous material of wall, or in case of an increased air gap, the safety factor was assumed. It was decided, that the value of this factor will be equal $x=2$. Thus, the postulated attraction force of the gripper will be equal $\mathrm{F}=3200 \mathrm{~N}$, with an air gap of $\delta=0.2 \mathrm{~mm}$. Figure 1 presents the algorithm of design (a), geometric dimensions (b), and the magnetic circuit (c) of the designed by authors axis symmetric electromagnetic gripping system, supplied with 24 DC voltage.

The designed electromagnetic gripper was imported to Comsol environment as a *dxf file. To analyze thermal phenomena and to check the correctness of developed model, the AC/DC module, including Magnetic Fields, Heat Transfer and Multiphysics, was selected. In 3D analyzing of the temperature distribution in the tested object, it is necessary to create additional geometry, which have physical properties of the medium in which the tested system is placed in. In this case the medium is air at temperature of $20{ }^{\circ} \mathrm{C}$. After assigning material properties to all domains, the power sources were assigned, that is power losses generated by the current flowing in the winding and power losses in the core of gripper. Then the model was discretized with four-sided elements. The developed 3D field model of the gripper enables the analysis of electromagnetic and thermal phenomena as well as the parametric analysis of the impact of current change in the winding, and the impact of the thickness of the air gap between the gripper and attracted surface on the value of the magnetic force.

\footnotetext{
Corresponding author: mariusz.baranski@put.poznan.pl
} 


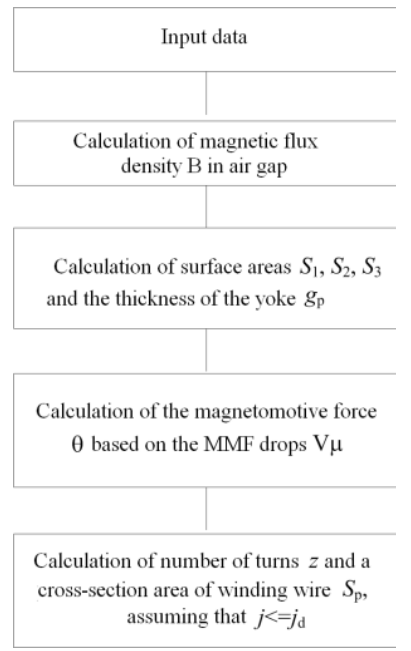

(a)

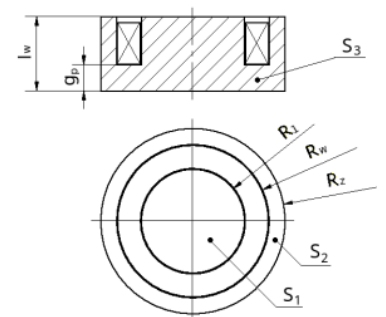

(b)

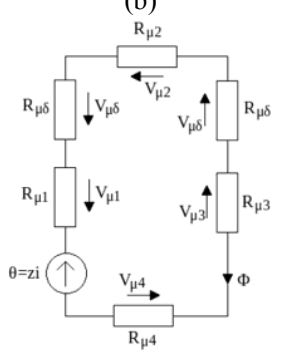

(c)
Fig. 1. Elaborated algorithm (a), geometric dimensions (b) and magnetic circuit (c) of the axis-symmetric gripper, where B magnetic flux density, S1 - surface area of the cylinder, S2 surface area of the ring, S3-surface area of the cross-section, $\mathrm{R} 1$-cylinder radius, $\mathrm{Rw}$ - inner ring radius, $\mathrm{Rz}$ - outer ring radius, gp- thickness of the base, lw- height of the gripper.

\section{Results of the simulation}

On the basis of the presented algorithm of DC gripper designing, the authors proposed the axis-symmetric electromagnetic gripping system. In order to verified the correctness of the gripper, the authors elaborated $3 \mathrm{D}$ field electromagnetic and thermal model by using Comsol Multiphysics environment. This model was used to analyze the influence of the current value in coil on temperature distribution. The gripper was supplied with a $24 \mathrm{DC}$ voltage, which corresponds to the nominal value of the current flowing in winging equal to $I_{\mathrm{N}}=0.494 \mathrm{~A}$. The tests were carried out in steady-state. Figures 2-4 show the distribution of temperature field inside the gripper, on the attracted surface, and in the air, in which the object is placed in, for following values of the coils' current: $0.2 \mathrm{~A}, 0.494 \mathrm{~A}$ and $1 \mathrm{~A}$.
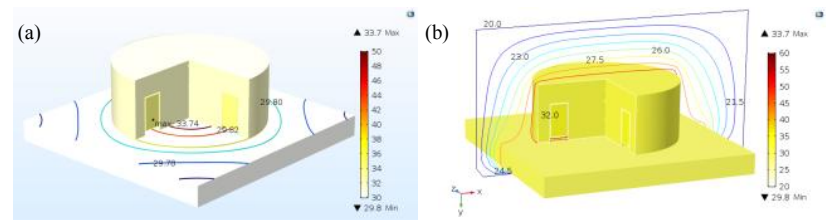

Fig. 2. Thermal field distribution of (a) gripper and attracted surface and (b) the air surrounding the object for current value of $\mathrm{I}=0.2 \mathrm{~A}$.
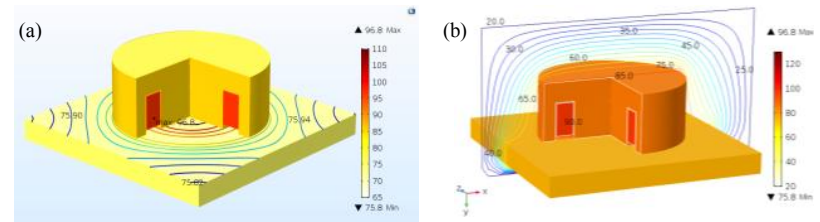

Fig. 3. Thermal field distribution of (a) gripper and attracted surface and (b) the air surrounding the object for current value of $I=I_{\mathrm{N}}=0.494$ A.
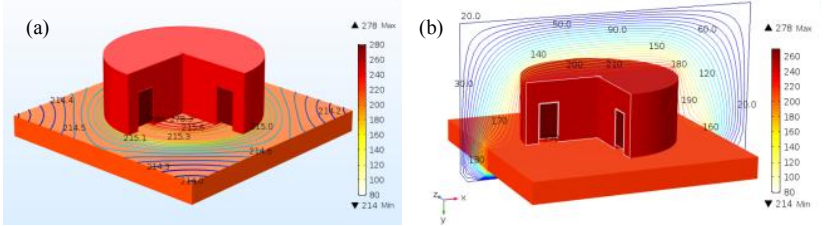

Fig. 4. Thermal field distribution of (a) gripper and attracted surface and (b) the air surrounding the object for current value of $I=1$ A.

\section{Summary}

The presented results of the simulation in designed gripper indicate the correctness of design calculations and the developed model. A strong influence of current changes in gripper winding on thermal field distribution in analyzed gripper was observed. Temperature of all gripper elements increases with the current of coil increases. When the gripper coil is supplied with the nominal current, the winding temperature does not exceed $98{ }^{\circ} \mathrm{C}$. This is a safe value for the windings designed in class $\mathrm{F}$ of insulation even with continuous operation of the device. For values of current greater than nominal, the coil exceeds the temperature-rise limit for $\mathrm{F}$ class isolation. On the basis of the above, it can be concluded that after exceeding the nominal operating current for the coil, the electro insulating system can be damaged.

\section{References}

1. J. Maempel, S. Koehring, C. Schilling, H. Witte: Using Different Adhesion Technologies in Modular Robot for Climbing, ISR / ROBOTIK, 1258-1264, (2010)

2. N. R. Kolhalkar and S. M. Patil: Wall Climbing Robots: A Review, International Journal of Engineering, 1, 5, 227 229,(2012)

3. M. Moniri, M. Bamdad, M. Sayyadan: A novel design of wall climbing robot for inspection of storage steel tanks, 2015 3rd RSI International Conference on Robotics and Mechatronics, 557-562, (2016)

4. H. Ishihara: Basic study on wall climbing robot with magnetic passive wheels, 2017 IEEE International Conference on Mechatronics and Automation, 1964-1969, (2007)

5. S. Yang: Thermal analysis of a DC electromagnet with high thermal conductivity inserts, Tallahassee: Florida State University, (2016)

6. Comsol Multiphysics 5.2a User's Guide, Modeling Guide and Model Library, Documentation Set, Comsol AB, (2019)

7. O.C. Zienkiewicz: Metoda elementów skończonych, Warszawa: Arkady, (1972).

8. C. Jiannan, H. Kai, F. Haitao, C. Hao, H. Shaojie, W. Zhou: The design of permanent-magnetic wheeled wallclimbing robot, IEEE International Conference on Information and Automation (ICIA), (2017)

9. Cengel Y.A: Heat transfer: a practical approach, Boston: McGraw-Hill, (2003)

10. M. Baranski, W. Szelag, C. Jedryczka: Influence of temperature on partial demagnetization of the permanent magnets during starting process of line start permanent magnet synchronous motor, Electrical Machines (SME), International Symposium on, Poland, (2017) 